\title{
A New Algorithm for Solving III-conditioned Linear System with an Auto- Parameter
}

\author{
Tianyu He ${ }^{a}$, Jinshan Wang ${ }^{b}$ and Zonghao Tian ${ }^{c}$ \\ Mathematics section, Army Officer Academy, Hefei 230031, China \\ atianyu_he@126.com, bhtyhit@sina.com, ctzh1109180769@163.com
}

Keywords: Particle swarm optimization algorithm, auto-parameter, ill-conditioned linear system.

\begin{abstract}
A new simple and effective method for solving ill-conditioned linear systems is presented in this paper. This method tried mainly not to decrease the error caused in direct solving, instead, it tries to transfer this error to a medium variable. At the same time, a parameter is introduced. In order to obtain the best parameter, PSO is used. An algorithm based on this method is presented, and examples show that this algorithm could solve extremely ill-conditioned linear systems correctly and stably.
\end{abstract}

\section{Introduction}

It is widely known that the solutions of linear systems of equations are sensitive to the round-off error. For some linear systems a small change in one of the values of the coefficient matrix or the right-hand side vector causes a large change in the solution vector. When the solution is highly sensitive to the values of the coefficient matrix $A$ or the right-hand side constant vector $\mathrm{b}$, the equations are said to be ill-conditioned. Therefore, we cannot easily rely on the solutions coming out of an ill-conditioned system.

Ill-conditioned systems pose particular problems when the coefficients are estimated form experimental results [1]. For a system with condition number $\kappa(A)=\|A\|\left\|A^{-1}\right\|$ on its coefficient matrix, one can expect a loss of roughly $\lg \kappa(A)$ decimal places in the accuracy of the solution [2]. There is a theorem about this in reference [3].

Theorem Let $A \in \mathbb{R}^{n \times n}$, det $A \neq 0, x$ and $x+\delta x$ satisfy the equation, respectively

$A x=b$

$(A+\delta A)(x+\delta x)=b+\delta b$

Here, $b \neq 0,\|\delta A\|$ satisfys

$$
\frac{\|\delta A\|}{\|A\|}<\frac{1}{\kappa(A)}
$$

Then

$$
\frac{\|\delta x\|}{\|x\|} \leq \frac{\kappa(A)}{1-\left\|A^{-1}\right\|\|\delta A\|}\left(\frac{\|\delta A\|}{\|A\|}+\frac{\|\delta b\|}{\|b\|}\right)
$$

In other words, solving very ill-conditioned linear systems by classical methods is not usual. It is difficult to get accurate results by classical methods.

\section{Error Transfer Method with Auto-Parameter}

\subsection{Algorithm Design.}

In the process of solving linear equations, we found that even if the solution of $A x=b$ is not accurate, the margin of error on both sides of the equation can be small. In other words, if $\tilde{x}$ is the solution of $A x=b$, which is obtained by using the conventional algorithm for solving linear equations. Though $\tilde{x}$ is not the accurate result, $\|A \tilde{x}-b\|$ can be very small. 
Assume $x^{*}$ as the accurate solution of $A x=b$. Aiming to get $x^{*}$, let's start by solving the equation below

$$
x^{*}=C \tilde{y}
$$

Here, $C$ is a non singular matrix of $n \times n$.

We know that even though $\tilde{y}$ is not the accurate result of equation (1), both sides of the equation (1) can be precise equal, and then we can get a comparatively accurate $x^{*}$ by calculating $C \tilde{y}$. Due to $x^{*}$ is not known, the equation (1) cannot be calculated directly. But we have the original equation $A x=b$, and the following equation is accurately established

$$
A x^{*}=b
$$

So (1) can be changed to

$$
A x^{*}=A C \tilde{y}=b
$$

By solving the equation (3), $\tilde{y}$ can be obtained. And then we take $\tilde{y}$ to the equation (1), the exact solution $x^{*}$ of $A x=b$ is obtained.

Because of the coefficient matrix is ill-conditioned, it will lead to great error. But this error is directly reflected in $\tilde{y}$, the effect on $x$ is very small. In reference [4] it is called error transfer method.

The selection of matrix $\mathrm{C}$ will directly affect the validity of the algorithm. If $C=I$, nothing has changed; If $C=\operatorname{diag}\left\{d_{1}^{-1}, d_{2}^{-1}, \cdots, d_{n}^{-1}\right\}$, here, $d=\sum_{i=1}^{n} \max \left(a_{i, j}\right)$, it's equal to the column equilibrium of the coefficient matrix.

In this paper, let $C=\omega A^{T}, \omega$ is a parameter, it can be obtained by using modern intelligent optimization, such as particle swarm optimization algorithm, then the formula (3) is transformed into

$$
A x^{*}=b=A \omega A^{T} \tilde{y}
$$

Then, using Gaussian Elimination to solve $A \omega A^{T} y=b$, and $\tilde{y}$ as the solution can be obtained, wherein, $\tilde{y}$ contains the parameters $\omega$. In order to find the optimal parameter $\omega$, we're trying to minimize the remainder. Here, let

$$
F(\omega)=\left\|A \omega A^{T} \tilde{y}-b\right\|
$$

The optimal parameters $\omega^{*}=\arg \min F(\omega)$. In order to obtain $\omega^{*}$, PSO can be used.

\subsection{Particle Swarm Optimization Algorithm.}

Particle swarm optimization algorithm (PSO) is used to simulate the birds foraging process, and every bird, according to its current position, the optimal location where they went, and the optimal position of the birds, updates their speed and position, and finally search the global optimal solution, and the standard PSO is commonly used now[5].

The particle swarm algorithm uses the following formula to update the location of the particle:

$$
\begin{aligned}
& v_{i}^{d}=\omega \bullet v_{i}^{d}+c_{1} \bullet \operatorname{rand}_{1} \bullet\left(\text { pbest }_{i}^{d}-x_{i}^{d}\right)+c_{2} \bullet \operatorname{rand}_{2} \cdot\left(\text { Gbest }_{i}^{d}-x_{i}^{d}\right) \\
& x_{i}^{d}=x_{i}^{d}+\alpha v_{i}^{d}
\end{aligned}
$$

Wherein, $i=1,2, \cdots, m ; d=1,2, \cdots, D ; \omega \geq 0, \quad D$ is the dimension of the search space, $X_{i}=\left(x_{i}^{1}, x_{i}^{2}, \cdots, x_{i}^{D}\right)$ is the position of the NO. $i$ particle, $V_{i}=\left(v_{i}^{1}, v_{i}^{2}, \cdots, v_{i}^{D}\right)$ is the speed of the NO. $i$ particle. pbest $_{i}=\left(\right.$ pbest $_{i}^{1}$, pbest $_{i}^{2}, \cdots$, pbest $\left._{i}^{D}\right)$ is the optimum location of a particle, Gbest $_{i}=\left(\right.$ Gbest $_{i}^{1}$, Gbest $_{i}^{2}, \cdots$, Gbest $\left._{i}^{D}\right)$ is the optimum location of the populations.

The choice of PSO parameters can have a large impact on optimization performance. Trelea suggests that $\omega=0.6 ; c_{1}=c_{2}=1.7$, but Eberhart suggests that $\omega=0.729 ; c_{1}=c_{2}=1.494$. In this paper we take Trelea's suggestion.

\subsection{Algorithm Steps}

The steps of using error transfer method with auto-parameter to solve $A x=b$ are as follows: 
Step1: Using Gaussian Elimination to solve $A \omega A^{T} y=b$, then $\tilde{y}$ as the solution can be obtained;

Step2: Let $F(\omega)=\left\|A \omega A^{T} \tilde{y}-b\right\|$, using Particle Swarm Optimization to obtain the $\omega^{*}=\arg \min F(\omega)$;

Step3: then $x^{*}=\omega^{*} A^{T} \tilde{y}$, is the solution of $A x=b$.

\section{Computational Tests.}

Test Problem. Consider the linear system $H x=b$.

Eg.1 Wherein $H$ is the Hilbert matrix defined by $H_{N \times N}=\left(h_{i, j}=\frac{1}{i+j-1}\right)$.

Eg.2 Wherein $H$ is the Pascal matrix defined by $H_{N \times N}=\left(h_{i, j}\right), h_{j, 1}=h_{1, j}=1, j=1,2, \cdots, n$, $h_{i, j}=h_{i-1, j}+h_{i, j-1}, i, j=2,3, \cdots, n$.

Eg.3 $H_{N \times N}=\left(h_{i, j}\right), h_{i, j}=\max \{i, j\}, i, j=1,2, \cdots, n$.

The right hand side vector of the equation above will be considered as $b=\left(b_{i}=\sum_{j=1}^{n} a_{i j}\right)$ and $b=\left(b_{i}=\sum_{j=1}^{n} a_{i j} \times j\right)$, obviously, the exact solution of the equations are $x=(1,1, \cdots, 1)^{T}$ and $x=(1,2, \cdots, \mathrm{n})^{T}$.

Here, we take 3 methods to solve the test problem. Method 1: Gaussian Elimination; Method 2 :

Error transfer method; Method 3: Error transfer method with auto-parameter。 Table 2 and table 3 respectively show the results of the test 1 and the precision of test 2 .

Table 1 simply shows that $H$ is getting ill-conditioned by increasing the dimension of the matrix, and subsequently the system cannot be solved easily. Note that the condition numbers in Table 1 have been obtained using MATLAB.

Table 2 shows the method 1 (Gaussian Elimination) is powerless for solving ill-conditioned linear system, but the method 3 (Error transfer method with auto-parameter) has strong ability to solve ill-conditioned problems. Results of method 3 are very close to the exact solution. The results were satisfactory. That is to say the method which is proposed in this paper is effective in solving ill-conditioned linear system.

From the table 3, we can see that the method 2 and method 3 have good calculation results whether in good-conditioned linear system or in ill-conditioned linear system. The accuracy of the calculation is the same when the coefficient matrix is good-conditioned. As the condition number of the coefficient matrix increase on, method 3 performs better than method2. That is to say method 3 performs better when the coefficient matrix is in extremely ill-conditioned, it has higher precision. But, obviously, the process of method 3 takes more time.

Table 1. The condition number of different Hilbert matrices

\begin{tabular}{|c|c|c|c|}
\hline Order of matrix & 10 & 15 & 20 \\
\hline $\begin{array}{c}\text { Condition number of } \\
\text { Hilbert matrix }\end{array}$ & $1.6025 \times 10^{13}$ & $2.6388 \times 10^{17}$ & $2.5072 \times 10^{18}$ \\
\hline $\begin{array}{c}\text { Condition number of } \\
\text { Pascal matrix }\end{array}$ & $4.1552 \times 10^{9}$ & $2.8399 \times 10^{15}$ & $1.3533 \times 10^{21}$ \\
\hline
\end{tabular}


Table 2. Results of test 1

\begin{tabular}{|c|c|c|c|c|}
\hline \multirow{2}{*}{$\begin{array}{c}\text { Eg.1 } \\
n=10\end{array}$} & \multicolumn{2}{|c|}{ Method 1 } & \multicolumn{2}{c|}{ Mehtod 3 } \\
\cline { 2 - 5 } & $x_{i}=1$ & $x_{i}=i$ & $x_{i}=1$ & $x_{i}=i$ \\
\hline$x_{1}$ & 1.0000000319 & 0.9999995899 & 1.0000006924 & 0.9999972887 \\
\hline$x_{2}$ & 0.9999947886 & 2.0000636454 & 0.9999771009 & 2.0001260053 \\
\hline$x_{3}$ & 1.0002072102 & 2.9975816218 & 1.0001676046 & 2.9986496960 \\
\hline$x_{4}$ & 0.9964682232 & 4.0391564866 & 0.9996069238 & 4.0052817888 \\
\hline$x_{5}$ & 1.0321920676 & 4.6678661708 & 1.0001454835 & 4.9927193102 \\
\hline$x_{6}$ & 0.8250827692 & 7.6013977243 & 1.0003186757 & 5.9990412315 \\
\hline$x_{7}$ & 1.5964067004 & 2.7100588331 & 1.0000936404 & 7.0056899499 \\
\hline$x_{8}$ & -0.2825082305 & 12.3392565478 & 0.9998154594 & 8.0042394100 \\
\hline$x_{9}$ & 2.6169566071 & 19.039594943 & 0.9997052590 & 8.9983947378 \\
\hline$x_{10}$ & 0.2528157491 & -35.973094891 & 0.9998017086 & 9.9943240293 \\
\hline
\end{tabular}

Table 3. Precision of test 2

\begin{tabular}{|c|c|c|c|c|c|c|c|}
\hline \multicolumn{2}{|c|}{} & \multicolumn{2}{|c|}{ Eg.1 } & \multicolumn{2}{c|}{ Eg.2 } & \multicolumn{2}{c|}{ Eg.3 } \\
\hline \multirow{2}{*}{ Method } & $\begin{array}{c}\text { Order of } \\
\text { matrix }\end{array}$ & $x_{i}=1$ & $x_{i}=i$ & $x_{i}=1$ & $x_{i}=i$ & $x_{i}=1$ & $x_{i}=i$ \\
\hline \multirow{3}{*}{ Method 2 } & 20 & $7 \sim 8$ & 7 & $8 \sim 9$ & $7 \sim 8$ & $13 \sim 14$ & $12 \sim 13$ \\
\cline { 2 - 8 } & 60 & $6 \sim 7$ & 6 & $8 \sim 9$ & $6 \sim 7$ & $11 \sim 12$ & $10 \sim 11$ \\
\cline { 2 - 8 } & 100 & $7 \sim 8$ & 6 & $8 \sim 9$ & 7 & $10 \sim 12$ & 10 \\
\hline \multirow{3}{*}{ Method 3 } & 20 & $7 \sim 8$ & 7 & $8 \sim 9$ & $7 \sim 8$ & $13 \sim 14$ & $12 \sim 13$ \\
\cline { 2 - 8 } & 60 & $7 \sim 8$ & $6 \sim 7$ & $8 \sim 9$ & $7 \sim 8$ & $11 \sim 12$ & $10 \sim 11$ \\
\cline { 2 - 8 } & 100 & $7 \sim 8$ & $6 \sim 7$ & $8 \sim 9$ & 8 & $10 \sim 12$ & 10 \\
\hline
\end{tabular}

\section{Summary}

In this article, we have developed a direct method-error transfer method. We introduce a parameter in order to make a better matrix of the intermediate process in solving ill-conditioned linear system. And give a method for obtaining optimal parameters by PSO. Numerical tests show the method is useful. The method can be spread to other areas such as engineering.

\section{References}

[1]. C.-S. Liu, W. Yeih, S.N. Atluri, On solving the ill-conditioned system $A x=b$ : General-purpose conditioners obtained from the boundary-cllocation solution of the Laplace wquation, using Trefftz expansions with multiple length scales, CMES Comput. Model. Eng. Sci. 44(2009), 281-311.

[2]. Fazlollah Soleymani, A new method for solving ill-conditioned linear systems, Opuscula Math.

[3]. Q.Y. Li, Z. Guan, F.S. Bai, Principle of numerical calculation, Tsinghua university press, Beijing, 2000, pp. 166-168.

[4]. S.R. Hu, X.W. Luo, A new method for solving ill-conditioned linear system: error transfer method. Journal of South China Agricultural University. 22(2001), 92-94.

[5]. J. Zhuo, The application of MATLAB in mathematical modeling, Beihang University Press, Beijing, 2014, pp. 150-163. 